\title{
Descripción clínico-epidemiológica de pacientes con enfermedad inflamatoria intestinal en una clínica de cuarto nivel en Cali
}

\section{Clinical-epidemiological description of patients with inflammatory bowel disease in a fourth level clinic in Cali}

Carlos A. Rojas, MD, ${ }^{*}$ Santiago Sánchez-Londoño, MD, ${ }^{2}$ Nelson E. Rojas, ${ }^{3}$ Mauricio Sepúlveda, MD, ${ }^{1}$ Jairo García, MD, ${ }^{1}$ Diego Jiménez, MD, ${ }^{1}$ Catalina Maldonado, MD, ${ }^{1}$ Angélica Tobón, MD. ${ }^{2}$

Médico internista y gastroenterólogo de la Fundación Valle del Lili y docente de medicina interna y gastroenterología de la Universidad ICESI. Cali, Colombia.

Residente de medicina interna, Universidad ICESI. Cali, Colombia.

${ }^{3}$ Estudiante de medicina, Universidad ICESI. Call, Colombia.

Datos presentados en póster el 5 y 6 de abril de 2019 en el Congreso de la Pan American Crohn's and Colitis Organization. Cartagena, Colombia.

*Correspondencia: Carlos A. Rojas, MD, croj016@yahoo.com

Fecha recibido: $27 / 05 / 19$ Fecha aceptado: $15 / 10 / 19$

\section{Resumen}

Introducción: en América Latina, la enfermedad inflamatoria intestinal (EII) es poco frecuente y la información, limitada. Se describieron características de los pacientes con Ell en una unidad de gastroenterología de una clínica de alto nivel de atención en Cali, Colombia. Materiales y métodos: estudio descriptivo de pacientes que consultaron con diagnóstico de enfermedad de Crohn (EC) o colitis ulcerativa (CU) a la Clínica Fundación Valle del Lili entre enero de 2011 y diciembre de 2015. Se realizó un análisis con Statistical Package for the Social Sciences de IBM (SPSS) versión 19, se calcularon la mediana y el rango intercuartílico para las variables numéricas, y frecuencias para las variables cualitativas. Resultados: se incluyeron 416 participantes, 115 con EC y 301 con CU. El $41 \%$ se clasificó como enfermedad leve, 23,5\% moderada y 35,3\% grave. Se realizó cirugía a 24 pacientes $(9,0 \%)$ con CU y $53(46,1 \%)$ con EC. En esta última, el manejo más frecuente fue los medicamentos biológicos (32,2\%), seguidos de inmunomoduladores $(27,8 \%)$, esteroides (20\%) y ácido 5-aminosalicílico (5-ASA) (11,3\%). El tratamiento más frecuente de CU fue 5-ASA (84,8 \%), seguido de esteroides (32,19\%), azatioprina (24,6\%) y biológicos (15,9\%). Conclusiones: el diagnóstico precoz sigue siendo un reto. La gravedad de la CU en los pacientes estudiados fue menor a la reportada en el mundo, lo cual no ocurrió con los pacientes con EC y podría estar en relación con el retraso diagnóstico. El uso de medicamentos biológicos se acercó a lo reportado en el primer mundo. Es difícil determinar si las menores tasas de cirugía se deben a una mejor respuesta clínica o a un difícil acceso a estas intervenciones.

\section{Palabras clave}

Enfermedades inflamatorias del intestino, colitis ulcerativa, enfermedad de Crohn.

\begin{abstract}
Objectives: In Latin America, inflammatory bowel disease (IBD) is rare, and information about it is limited. This article describes characteristics of IBD patients in a gastroenterology unit at a high-level clinic in Cali, Colombia. Materials and methods: This is a descriptive study of patients diagnosed with Crohn's disease (CD) or ulcerative colitis (UC) at the Clínica Fundación Valle de Lili between January 2011 and December 2015. Statistical analysis was performed with SPSS version 19. Medians and interquartile ranges were calculated for numerical variables. Frequencies were calculated for qualitative variables. Results: The 416 participants included 115 with CD and 301 with UC. Of the total cases, $41 \%$ were classified as mild, $23.5 \%$ as moderate and $35.3 \%$ as severe. Surgery was performed in 24 patients $(9.0 \%)$ with UC and $53(46.1 \%)$ with $\mathrm{CD}$. CD was most frequently managed with biologicals (32.2\%), followed by immunomodulators $(27.8 \%)$, steroids $(20 \%)$ and $5-A S A(11.3 \%)$. The most frequent treatment for UC was $5-A S A(84.8 \%)$, but $32.19 \%$ received steroids, $24.6 \%$ received azathioprine and $15.9 \%$ received biologicals. Conclusions: Early diagnosis remains a challenge. The severity of UC but not CD in the patients studied was less than that reported elsewhere in the world. The difference could be related to diagnostic delay. The use of biologicals was close to that reported in the first world. It is difficult to determine if lower surgery rates are due to better clinical response or to difficult access to these interventions.
\end{abstract}

\section{Keywords}

Inflammatory bowel diseases, ulcerative colitis, Crohn's disease. 


\section{INTRODUCCIÓN}

La enfermedad de Crohn (EC) y la colitis ulcerativa (CU) son afecciones crónicas infrecuentes del tracto gastrointestinal cuya etiología es solo parcialmente comprendida. Su diagnóstico es complejo y a menudo se retrasa debido a la baja incidencia y la baja sospecha clínica. La EC afecta el tracto gastrointestinal de forma transmural desde la boca hasta el ano, mientras que la CU afecta la mucosa colónica.

Varios estudios han evaluado la epidemiología de la enfermedad inflamatoria intestinal (EII) en varias regiones. En América del Norte, la incidencia varía de 2,2 a 19,2 casos por 100000 años-persona para CU y de 3,1 a 20,2 casos por 100000 años-persona para EC (1). En América Latina, un estudio argentino encontró una incidencia de EII de 2,2 por 100000 habitantes; mientras que un estudio chileno no estableció incidencia, pero sí una mayor proporción de casos de CU y características similares a las reportadas en Europa (2). En general, a medida que los países en desarrollo elevan su nivel de vida, también aumenta la incidencia de EII (3).

La información disponible en Colombia es limitada. La primera serie de casos se publicó en 1991 (4), un estudio más reciente encontró que la $\mathrm{CU}$ tiene un comportamiento relativamente benigno con bajas tasas de cirugía y mortalidad, aunque la tendencia es a un mayor diagnóstico de EC por su gravedad en nuestra población (5), estos datos confirmaron un estudio más pequeño (6). En el suroccidente colombiano no hay estudios hasta el momento. Con este trabajo se busca describir las características demográficas y clínicas de los pacientes con EII de la Clínica Fundación Valle del Lili.

\section{MATERIALES Y MÉTODOS}

Se realizó un estudio descriptivo con recolección retrospectiva de la información. Se incluyeron pacientes que consultaron a la Fundación Valle del Lili con diagnóstico de la clasificación internacional de enfermedades-10. ${ }^{a}$ edición (CIE-10) de EC y CU desde enero de 2011 hasta diciembre de 2015. Se preseleccionaron los pacientes a través de la revisión manual de las historias clínicas en el sistema de documentación informática de la Fundación Valle del Lili (SAP) que tuvieran diagnósticos de EC y CU por códigos del CIE-10 entre enero de 2011 y diciembre de 2015. Se incluyeron todos los pacientes con edad mayor o igual a 18 años de ambos sexos que tuvieran diagnóstico de $\mathrm{EC} \mathrm{y} \mathrm{CU}$ por el cumplimiento de criterios clínicos, séricos, histológicos o endoscópicos, y que hayan acudido a la consulta externa de gastroenterología entre enero de 2011 y diciembre de 2015. Se estimó que se atenderían 100 pacientes anuales aproximadamente. Se excluyeron los pacientes menores de 18 años y quienes, a pesar de tener asignado un código CIE-10 para EII, no cumplían criterios clínicos, séricos, histológicos o endoscópicos para el diagnóstico claro de la enfermedad.

En la base de datos del Centro de Investigaciones Clínicas de la Fundación Valle del Lili (BD Clinic) se recolectó la siguiente información discriminada por diagnóstico para su posterior análisis:

- Características del paciente: fecha y edad del diagnóstico, sexo, tipo de EII, tiempo de evolución desde la impresión diagnóstica hasta el ingreso a la primera consulta de gastroenterología, hospitalizaciones asociadas, comorbilidades, antecedente de tabaquismo o consumo de licor.

- Características de la enfermedad: cirugías relacionadas con EII; antecedente de EII en sus familiares de primer y segundo grado; presencia de autoanticuerpos positivos de cualquier tipo: los anticuerpos anticitoplasma de neutrófilo (ANCA), ANCA de tinción perinuclear ( $\mathrm{p}$-ANCA), ANCA de tinción citoplasmática (c-ANCA); valor de la albúmina y de la hemoglobina; y el tipo de tratamiento que recibe y recibió el paciente.

- Características de la CU: gravedad inicial de acuerdo con la Clasificación de Montreal (7); presencia o no de manifestaciones extraintestinales y remisión, definida como la resolución completa de los síntomas y sanación clínica, endoscópica o histológica de la mucosa colónica.

- Características de la EC: Clasificación de Montreal de la EC (7), clasificación Crohn Disease Activity Index (CDAI) (8) en el momento del diagnóstico, presencia o no de enfermedad fistulizante, presencia o no de manifestaciones extraintestinales, respuesta adecuada (definida como una mejoría de más de 100 puntos en el CDAI respecto a previo), recaída (definida como el incremento en los sintomas relacionados con su EII, que requirieron consulta médica y que condujo a un aumento en la dosis de tratamiento médico que venía recibiendo el paciente, o la introducción de una nueva medicación o cirugía). Se definió recaída temprana como menos de tres meses de haber logrado una remisión con una nueva aparición de síntomas. Se definió refractariedad a esteroides como el requerimiento de dosis de esteroides mayores a 0,75 $\mathrm{mg} / \mathrm{kg} /$ día de prednisolona al día después de 4 semanas de inicio de tratamiento. Se registraron las recurrencias según los criterios de Rutgeerts (9) y se registraron los hallazgos endoscópicos e histológicos según lo explícitamente indicado en la historia clínica.

Los estudios histopatológicos se consideraron positivos si mencionaban el diagnóstico explícitamente en el reporte o si informaban de alguna característica considerada como típica, basados en las definiciones histológicas del Consenso Europeo sobre la histopatología de la EII (10). 
Se realizó un análisis descriptivo de los datos registrados en BD Clinic con la herramienta Statistical Package for the Social Sciences de IBM (SPSS) versión 19, se calcularon la mediana y el rango intercuartílico (RIC) para las variables numéricas, mientras que las variables cualitativas se describieron con frecuencias. Al tratarse de un análisis retrospectivo de revisión de historias clínicas, las variables no registradas se reportaron como sin dato en los resultados y se excluyeron de los análisis por variables.

\section{RESULTADOS}

Se incluyeron 416 participantes: 115 con EC y 301 con CU $(27,6 \%$ y $72,4 \%)$, con una relación de 2,61:1 y mayor incidencia de CU; la mediana de edad en los primeros fue de 43 años (RIC $95 \%$ : 29-57) y para los pacientes con CU de 42 (RIC: 30-55). La mayoría eran mujeres, con similar frecuencia en la presencia de comorbilidades. Menos del $10 \%$ informó consumo de tabaco o alcohol. El antecedente familiar de EII fue más frecuente en EC. El tiempo promedio entre la primera consulta y el diagnóstico fue de 9,29 meses (RIC: 1-36 meses) para EC y 6 meses (RIC: 2-18 meses) para CU. En total, 105 pacientes $(32,5 \%)$ fueron hospitalizados en salas generales a causa de su enfermedad y $38(9,1 \%)$ fueron hospitalizados alguna vez en la unidad de cuidados intensivos (UCI); los pacientes con EC tuvieron mayor tendencia a la hospitalización (Tabla $\mathbf{1}$ ).

Tabla 1. Características de la población de estudio y hospitalizaciones

\begin{tabular}{lccc}
\multicolumn{1}{c}{ Características } & EC & CU & Total \\
& $\mathbf{N}(\%)$ & $\mathbf{N}(\%)$ & $\mathbf{N}(\%)$ \\
\hline Sexo & & & \\
- Femenino & $60(52,2)$ & $167(55,5)$ & $227(54,6)$ \\
- Masculino & $55(47,8)$ & $134(44,5)$ & $189(45,4)$ \\
Tabaquismo & $9(7,8)$ & $24(9,1)$ & $33(8,7)$ \\
- SD & 0 & 38 & 38 \\
Alcohol & $10(8,7)$ & $18(6,8)$ & $28(7,4)$ \\
- SD & 0 & 37 & 37 \\
Comorbilidades & $32(27,8)$ & $112(43,4)$ & $144(38,6)$ \\
- SD & 0 & 43 & 43 \\
Antecedente de Ella & $12(10,4)$ & $10(3,8)$ & $22(5,8)$ \\
- SD & 0 & 39 & 39 \\
Hospitalizaciones & $\mathrm{N}=115$ & $\mathrm{~N}=301$ & $\mathrm{~N}=416$ \\
- Ninguna & & & \\
- 1 & $22(33,8)$ & $196(76)$ & $218(67,5)$ \\
- 2 & $18(27,7)$ & $42(16,3)$ & $60(18,6)$ \\
- 3 o más & $17(26,2)$ & $14(5,4)$ & $31(9,6)$ \\
- SD & $8(12,3)$ & $6(2,3)$ & $14(4,3)$ \\
- Hospitalizaciones en sala general & $43(37,4)$ & $62(20,6)$ & $105(25,2)$ \\
- Hospitalizaciones en UCl & $17(14,8)$ & $21(7,0)$ & $38(9,1)$
\end{tabular}

${ }^{a}$ De EII en familiares de primer grado. SD: sin dato.
La mayoría de pacientes $(94,4 \%)$ no tuvo anticuerpos positivos. El valor promedio de la hemoglobina en los pacientes con EC fue 12,3 g/dL (RIC: 6,7-16,9) y en los pacientes con CU fue $13 \mathrm{~g} / \mathrm{dL}$ (RIC: 11-14). Las muestras histopatológicas se reportaron como positivas en 67 pacientes con EC $(58,3 \%)$ y en 200 pacientes con CU $(88,1 \%)$ (Tabla 2).

Tabla 2. Hallazgos histológicos y de laboratorio

\begin{tabular}{lccc|} 
& $\begin{array}{c}\text { EC } \\
\mathbf{N}(\%)\end{array}$ & $\begin{array}{c}\text { CU } \\
\mathbf{N}(\%)\end{array}$ & $\begin{array}{c}\text { Total } \\
\mathbf{N}(\%)\end{array}$ \\
\hline Histológicos & & & \\
- Negativos & $48(41,7)$ & $27(11,9)$ & $75(21,9)$ \\
- Positivos & $67(58,3)$ & $200(88,1)$ & $267(78,1)$ \\
- SD & 0 & 74 & 74 \\
Anticuerpos & & & \\
- Negativos & $108(93,9)$ & $250(94,7)$ & $358(94,4)$ \\
- ANCA & $3(2,6)$ & $3(1,1)$ & $6(1,6)$ \\
- p- ANCA & $0(0)$ & $1(0,4)$ & $1(0,3)$ \\
- ANCA & $0(0)$ & $3(1,1)$ & $3(0,8)$ \\
- ANA & $4(3,5)$ & $7(2,7)$ & $11(2,9)$ \\
- SD & 0 & 37 & 37
\end{tabular}

Según la Clasificación de Montreal, 114 de los pacientes con CU $(53,3 \%)$ se clasificaron como proctitis ulcerativa, $43(20,1 \%)$ como colitis izquierda y $57(26,6 \%)$ como pancolitis. En los pacientes con EC, la localización más común fue la ileal (54\%) (Figura 1). La gravedad de la $\mathrm{CU}$ en la consulta inicial se registró como asintomática en 23 pacientes $(10,7 \%)$, leve en $105(49,1 \%)$, moderada en $55(25,7 \%)$ y grave en $31(14,5 \%)$. Casi la mitad de los pacientes con EC $(48,6 \%)$ tuvo un comportamiento no estenosante y no penetrante, 2 pacientes $(1,7 \%)$ tuvieron enfermedad perianal, de los cuales uno se clasificó como B2 y el otro como B3; y 34 pacientes (40 \%) presentaron fístulas (Figura 2).

En EC, la manifestación clínica predominante fue el dolor abdominal, seguido de la diarrea. Según la clasificación CDAI, el $41 \%$ de los pacientes presentó enfermedad leve; el 23,5\%, enfermedad moderada; y el 35,29 \%, enfermedad grave (Tabla 3). Se encontraron manifestaciones extraintestinales en 28 pacientes con CU $(10,3 \%)$ y en 33 pacientes con EC ( $44 \%)$, en ambos casos la mayoría fueron articulares (Figura 3). La respuesta adecuada se encontró en 11 pacientes $(25,6 \%)$, mientras que fue inadecuada en 32 pacientes $(74,4 \%) ; 2(4,4 \%)$ presentaron recaída 


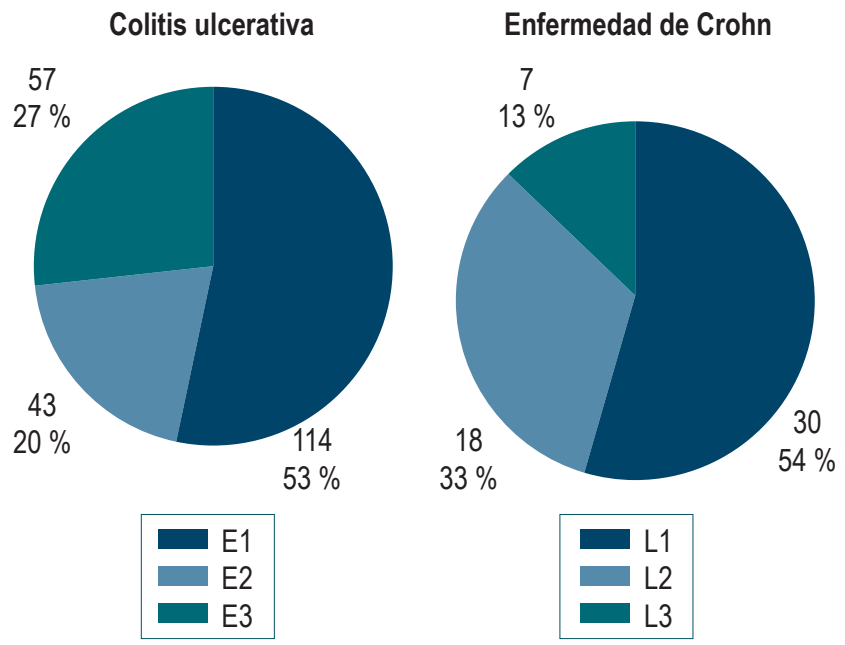

Figura 1. Extensión de la enfermedad según la clasificación de Montreal. E1: proctitis ulcerativa; E2: colitis izquierda; E3: pancolitis ulcerativa; L1: íleon terminal; L2: colon; L3: íleocolon. No se incluyó L4 por no haber pacientes con compromiso superior en nuestro estudio.
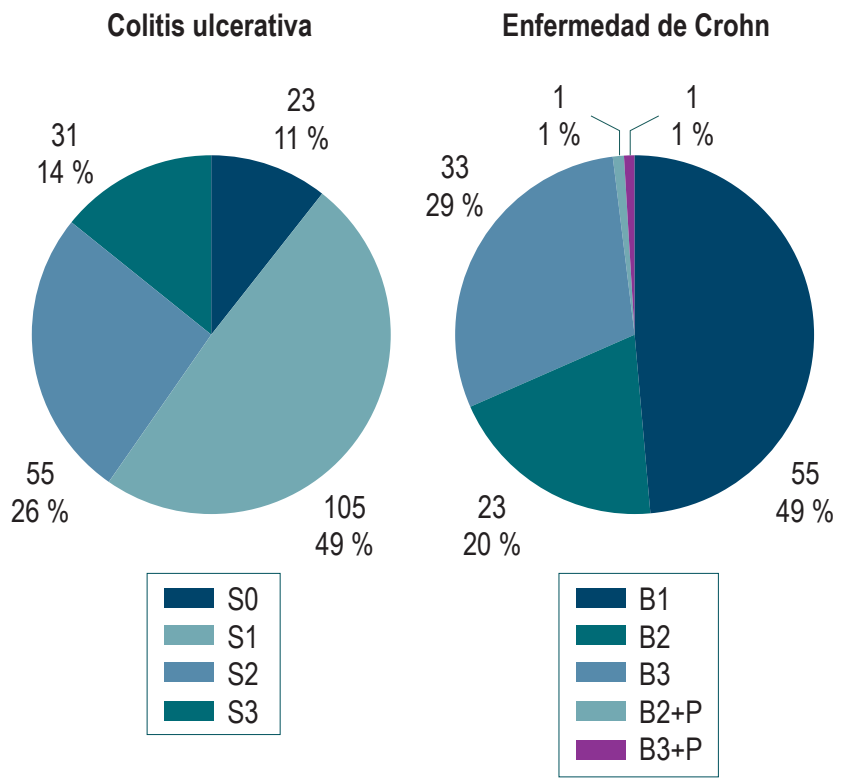

Figura 2. Severidad de la colitis ulcerativa y comportamiento de la enfermedad de Crohn según la clasificación de Montreal. S0: remisión clínica; S1: leve; S2: moderada; S3: severa; B1: no estenosante y no penetrante; B2: estenosante; B3: penetrante; P: perianal.

temprana y $8(17,4 \%)$ fueron refractarios al tratamiento. 24 pacientes con CU $(9,0 \%)$, y 53 con EC $(46,1 \%)$ fueron sometidos a algún tipo de cirugía relacionada con su diagnóstico de base; se realizó ilectomía en 11 pacientes con EC $(9,5 \%)$ y colectomía en $6(5,22 \%)$, sin reporte de recurrencias. La colectomía se realizó en 16 pacientes con
Tabla 3. Características de la EC

\begin{tabular}{|c|c|}
\hline Manifestaciones clínicas & $N(\%)$ \\
\hline Pérdida de peso & $12(10,4)$ \\
\hline Diarrea & $33(28,7)$ \\
\hline Dolor abdominal & $73(63,5)$ \\
\hline Anorexia & $2(1,7)$ \\
\hline Hematoquexia & $8(7,0)$ \\
\hline Edad al diagnóstico & $\mathbf{N}(\%)$ \\
\hline A1 & 0 \\
\hline A2 & $56(52,8)$ \\
\hline A3 & $50(47,2)$ \\
\hline SD & 9 \\
\hline CDAl inicio & $\mathrm{N}(\%)$ \\
\hline Remisión (< 150) & $14(41,2)$ \\
\hline Leve (150- 220) & $8(23,5)$ \\
\hline Moderado (220-450) & $12(35,3)$ \\
\hline Grave (> 450) & $0(0)$ \\
\hline SD & 81 \\
\hline
\end{tabular}

CU (6,67\%), siendo la intervención más frecuente. Solo 1 paciente con CU fue sometido a fistulectomía (4,16\%).

En cuanto al tratamiento médico de los pacientes con diagnóstico de EC, 23 (20\%) recibieron esteroides, 32 $(27,82 \%)$ azatioprina, $14(12,17 \%)$ derivados del ácido 5-aminosalicílico (5-ASA) y $37(32,17 \%)$ tratamiento con un medicamento biológico. De los pacientes con diagnóstico de CU, 85 (32,19\%) recibieron esteroides, 65 (24,62\%) azatioprina, $224(84,8 \%)$ derivados de 5-ASA y 42 (15,9\%) algún medicamento biológico (Figura 4).

\section{DISCUSIÓN}

Este estudio reunió 416 historias clínicas de pacientes con diagnóstico de EII en un centro de referencia de Cali y las evaluó retrospectivamente para conocer las características y la historia natural de estos pacientes. La proporción de pacientes con EC y CU (27,6\% frente a $72,3 \%)$ fue similar a lo reportado en las series colombianas por Juliao (15,8\% frente a $80,7 \%)(5)$, Yepes $(23,1 \%$ frente a $76,9 \%)(6)$ y Reyes $(24,2 \%$ frente a $75,8 \%)(11)$, así como las publicaciones en la literatura mundial $(12,13)$. Es llamativa la pro- 

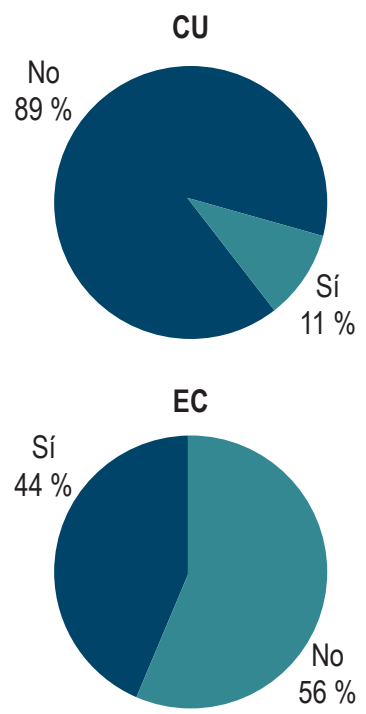

Colitis ulcerativa

Enfermedad de Crohn

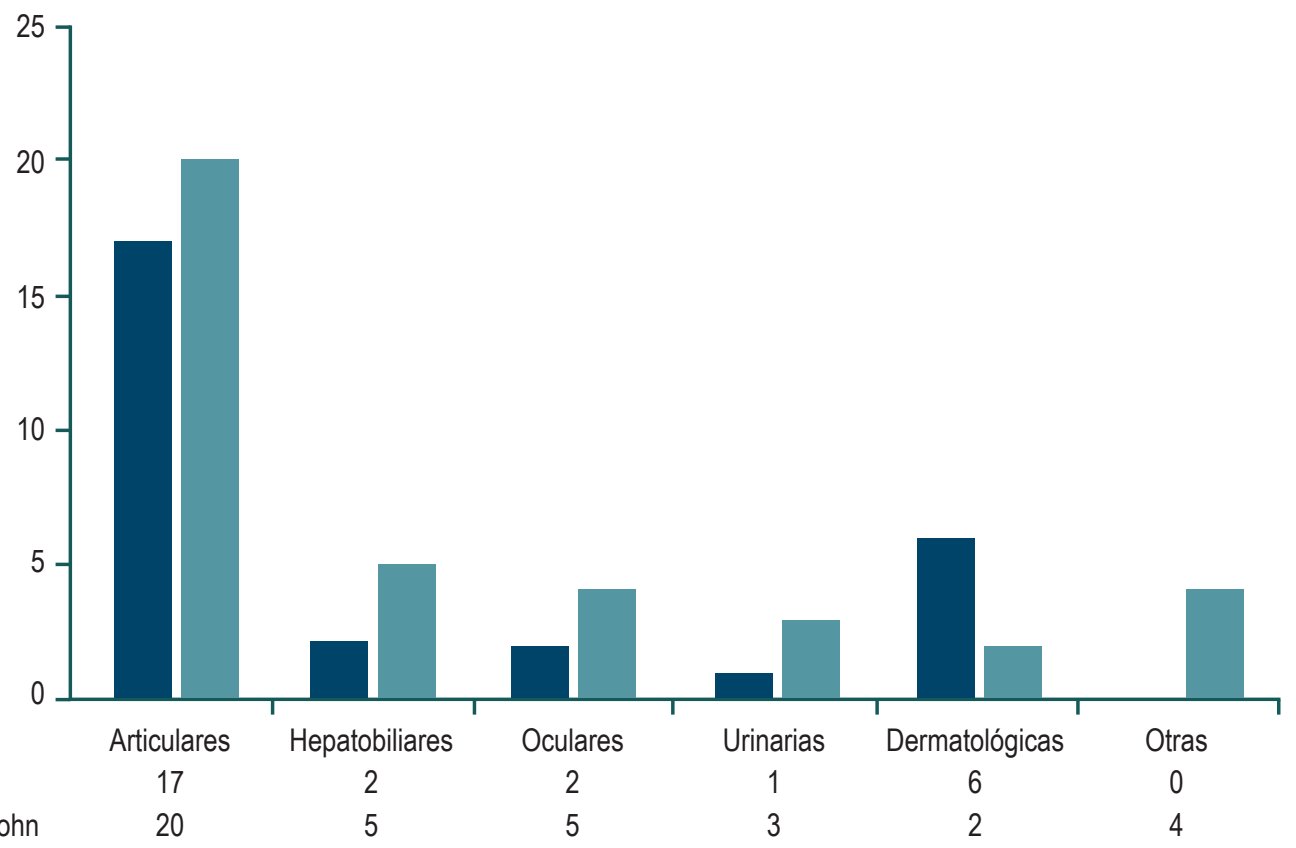

Figura 3. Manifestaciones extraintestinales. Los gráficos de sectores muestran la distribución de pacientes con y sin manifestaciones extraintestinales para colitis ulcerativa y enfermedad de Crohn. La distribución de cada tipo de manifestación se muestra en el gráfico de barras. CU: colitis ulcerativa; EC: enfermedad de Crohn.

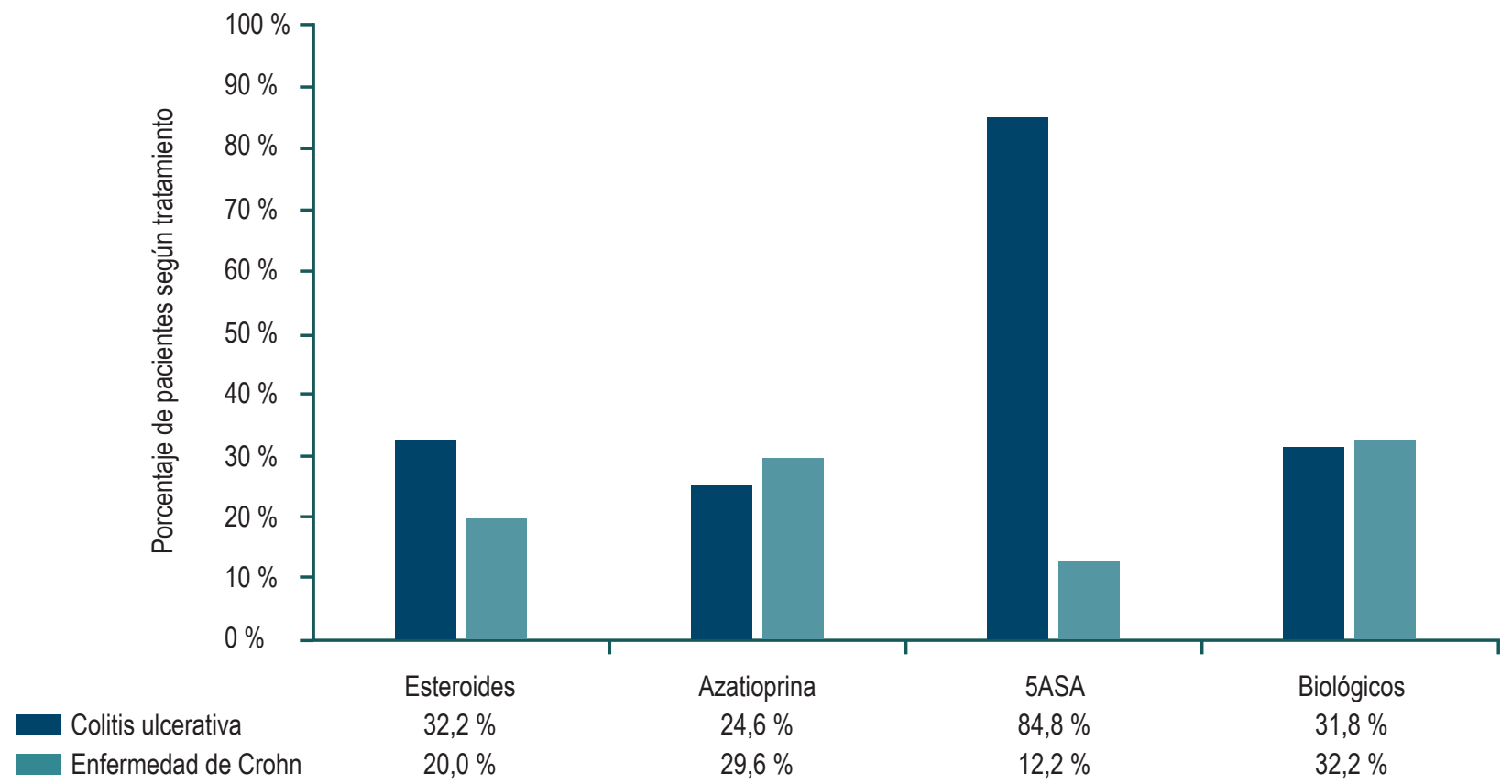

Figura 4. Tratamiento de los pacientes con colitis ulcerativa y enfermedad de Crohn.

porción con respecto a la primera serie colombiana de EII (9,3\% en EC frente a 90,7\% en CU) (14), consistente con las observaciones en países en los que la EII es una enfermedad emergente (6). En nuestro estudio, la mediana de edad al momento del diagnóstico fue similar a los reportes de Reyes (11) y otras series internacionales $(2,15,16)$.

De los 32 pacientes con EC y alguna comorbilidad $(27,8 \%)$, se presentaron $6(5,21 \%)$ con enfermedades 
oncológicas tanto sólidas como hematológicas, 5 (4,34 \%) con hipertensión arterial y $3(2,6 \%)$ con enfermedades reumatológicas. De los 112 pacientes con CU y comorbilidades $(43,4 \%)$, se registraron $30(11,6 \%)$ con hipertensión arterial (HTA), 18 (6,97\%) con enfermedades reumatológicas, $8(3,1 \%)$ con enfermedades oncológicas (de los cuales 2 reportaron cáncer de colon) y 7 (2,71\%) con enfermedades psiquiátricas (de los cuales 4 reportaron depresión). Fue llamativo que la prevalencia de trastornos psiquiátricos en nuestra población fue menor a la reportada en otras series $(17,18)$.

El antecedente de EII en algún familiar de primer grado estuvo presente en $10,4 \%$ de los pacientes con EC y $3,8 \%$ de los pacientes con CU. Un estudio danés estableció que la proporción de nuevos casos de EC con una historia familiar positiva alcanzó $12,2 \%$ y para CU fue de $8,8 \%$ (19). Curiosamente, un metaanálisis reportó una mayor frecuencia de antecedentes familiares en CU (12\%) que en EC (2\%) (20).

El comportamiento de la EC fue más grave que el de la CU en la mayoría de variables registradas en este trabajo. Más de la mitad de los pacientes de CU (53,3\%) tenía enfermedad limitada al recto y $26,6 \%$ pancolitis, lo cual va en línea con estudios colombianos previos $(5,6)$, pero difiere de estudios en el primer mundo, donde ha aumentado la frecuencia de pancolitis y en varios países es la manifestación más frecuente (21-23). Algo similar ocurre en los pacientes con EC, de los cuales solo el 12,7 \% tenía enfermedad ileocolónica, mientras que en la literatura se ha reportado una frecuencia cercana al $50 \%$. El 49,7 \% de los pacientes de CU tuvo enfermedad leve, el 25,7 \% moderada y el 14,5\% grave; en EC las frecuencias fueron de $41,17 \%, 23,5 \%$ y $35,3 \%$, respectivamente. El 10,7 \% de los pacientes con CU estaba asintomático, mientras que ningún paciente con EC estaba en remisión. La hospitalización tanto general como en la UCI fue más frecuente en los pacientes con EC que en aquellos con $\mathrm{CU}$; las manifestaciones extraintestinales también fueron más frecuentes en EC, lo cual coincide con la frecuencia reportada en la literatura.

En nuestro centro, el retraso diagnóstico fue mayor en EC que en CU (9,2 meses frente a 6,0 meses), lo cual es concordante con lo publicado en estudios nacionales, aunque mayores que los reportados en la literatura internacional. Un estudio multicéntrico italiano encontró un mayor retraso diagnóstico de meses en EC $(7,1)$ que en CU $(2,0)$, el cual fue mayor en una cohorte histórica que en una moderna (24). Un estudio surcoreano mostró mayores tasas de requerimiento quirúrgico en pacientes con mayores retrasos diagnósticos (25). La presencia de autoanticuerpos fue muy baja en comparación con los países con alta incidencia de EII, como Japón (26). En nuestro centro, el 58,3 \% de los pacientes con EC y el 88,1\% de los pacientes con CU tuvieron algún hallazgo histológico típico o fueron reportados como positivos para el diagnóstico en cuestión. La comparación de estos hallazgos con los de otros autores es difícil por la falta de estandarización en el reporte de especímenes histopatológicos de pacientes con EII. Un trabajo brasileño sugiere que el diagnóstico histopatológico estandarizado podría presentar un mayor porcentaje de diagnósticos concluyentes, especialmente en EC (27).

El uso de medicamentos biológicos en nuestros pacientes es similar al registrado en una serie reciente en la población colombiana, $35 \%$ para EC y $16 \%$ para CU (11), y parece que la tendencia es a aumentar su uso, como se demostró en un estudio económico en el que se reportó un aumento del uso de biológicos del 21,8 \% al 43,8 \% en EC y del 5,1 \% al 16,2 \% en CU entre 2007 y 2015 (28).

En nuestra población hubo una diferencia notable en los pacientes con EC y CU sometidos a cirugía abdominal, ya que $46,1 \%$ de los pacientes del primer grupo fue sometido a una intervención, en comparación con el 9,0 \% de los pacientes del segundo grupo. Incluso sin tener en cuenta la apendicectomía, el 33 \% de los pacientes con EC se sometió a cirugías abdominales. La cirugía más frecuentemente realizada, excluyendo la apendicectomía, fue la ilectomía en EC $(9,5 \%)$ y la colectomía en CU $(6,67 \%)$. La colectomía se realizó en el 5,22\% de los pacientes con EC. La frecuencia de intervención en nuestro estudio fue mayor a la reportada por Reyes $(27,5 \%$ frente a $4,8 \%)$ y similar a la publicada por Juliao ( $50 \%$ frente a $5,9 \%$ ) en la población colombiana, pero es baja en comparación con lo reportado en estudios con poblaciones de alta frecuencia, que oscila entre $37,9 \%$ y $50 \%(13,29)$ para EC y $8,3 \%$ y $32,4 \%$ para CU $(16,30)$. En este estudio no hubo reportes de recurrencia posquirúrgica a pesar de que la tasa reportada está alrededor del $80 \%$ (31).

La EII afecta cada vez más a la población colombiana y es imperativo conocer mejor la enfermedad y su comportamiento en nuestro medio. El diagnóstico temprano sigue siendo un reto muy importante, no solo para nuestro centro sino para todos los del país, especialmente en EC. La gravedad de la $\mathrm{CU}$ en nuestros pacientes fue menor a la reportada en el mundo, lo cual no ocurrió con los pacientes con EC y podría estar relacionado con el retraso diagnóstico. El uso de medicamentos biológicos se acerca cada vez más a lo reportado en el primer mundo, lo cual traerá beneficios clínicos y de calidad de vida a nuestros pacientes. Es difícil determinar si las menores tasas de cirugía se deben a una mejor respuesta clínica o a un peor acceso a estas intervenciones. Deben llevarse a cabo más estudios en nuestra población para continuar caracterizando el fenotipo de la enfermedad y su evolución a largo plazo, a medida que aumenta la incidencia en nuestro medio y se hacen disponibles nuevas opciones terapéuticas. 
Fuente de apoyo financiero

Ninguna.

\section{Conflictos de interés}

Ninguno.

\section{REFERENCIAS}

1. Molodecky NA, Soon IS, Rabi DM, Ghali WA, Ferris M, Chernoff G, Benchimol EI, Panaccione R, Ghosh S, Barkema HW, Kaplan GG.. Increasing incidence and prevalence of the inflammatory bowel diseases with time, based on systematic review. Gastroenterology. 2012;142(1):46-54.e42. https://doi.org/10.1053/j.gastro.2011.10.001

2. Figueroa CC, Quera PR, Valenzuela EJ, Jensen BC. Enfermedades inflamatorias intestinales: Experiencia de dos centros chilenos. Rev Med Chil. 2005;133(11):1295-304. https://doi.org/10.4067/S0034-98872005001100004

3. Kamm MA. Rapid changes in epidemiology of inflammatory bowel disease. Lancet. 2017;390(10114):2741-2. https://doi.org/10.1016/S0140-6736(17)32669-7

4. Lega-Siccar J, Lega LI. Colitis ulcerativa y enfermedad de Crohn del colon. Acta Médica Colomb. 1991;16(3):140-52.

5. Juliao-Baños F, Ruiz-Vélez MH, Flórez-Arango JF, DonadoGómez JH, Marín-Zuluaga JI, Monsalve-Arango C, JiménezGómez CA, Agudelo-Zapata Y, Velayos FS. Fenotipo e historia natural de la enfermedad inflamatoria intestinal en un centro de referencia en Medellín-Colombia. Rev Colomb Gastroenterol. 2010;25(3):240-51.

6. Yepes-Barreto IJ, Carmona R, Díaz F, Marín-Jiménez I. Prevalencia y características demográficas de la enfermedad inflamatoria intestinal en Cartagena, Colombia. Rev Colomb Gastroenterol. 2010;25(2):107-11.

7. Silverberg M, Satsangi J, Ahmad T. Toward an integrated clinical, molecular and serological classification of inflammatory bowel disease: Report of a Working Party of the 2005 Montreal World Congress of Gastroenterology. Can J Gastroenterol. 2005;19(Suppl A):5-36. https://doi.org/10.1155/2005/269076

8. Best W, Becktel J, Singleton J, Kern F. Development of a Crohn's disease activity index. National Cooperative Crohn's Disease Study. Gastroenterology. 1976;70(3):439-44. https://doi.org/10.1016/S0016-5085(76)80163-1

9. Rutgeerts P, Geboes K, Vantrappen G, Beyls J, Kerremans R, Hiele M. Predictability of the postoperative course of Crohn's disease. Gastroenterology. 1990;99(4):956-63. https://doi.org/10.1016/0016-5085(90)90613-6

10. Magro F, Langner C, Driessen A, Ensari A, Geboes K, Mantzaris GJ, Villanacci V, Becheanu G, Borralho Nunes P, Cathomas G, Fries W, Jouret-Mourin A, Mescoli C, de Petris G, Rubio CA, Shepherd NA, Vieth M, Eliakim R; European Society of Pathology (ESP); European Crohn's and Colitis Organisation (ECCO). European consensus on the histopathology of inflammatory bowel disease.
J Crohn's Colitis. 2013;7(10):827-51. https://doi.org/10.1016/j.crohns.2013.06.001

11. Reyes GA, Gil FL, Carvajal GD, Sánchez CB, Aponte DM, González CA, Gamba JI, Preciado JA, Marulanda JC, Sabbagh LC. Enfermedad inflamatoria intestinal: características de fenotipo y tratamiento en un hospital universitario de Bogotá. Rev Col Gastroenterol. 2018;117-26. https://doi.org/10.22516/25007440.196

12. Höie O, Wolters F, Riis L, Aamodt G, Solberg C, Bernklev T, Odes S, Mouzas IA, Beltrami M, Langholz E, Stockbrügger R, Vatn M, Moum B; European Collaborative Study Group of Inflammatory Bowel Disease (EC-IBD). Ulcerative colitis: Patient characteristics may predict $10-\mathrm{yr}$ disease recurrence in a European-wide population-based cohort. Am J Gastroenterol. 2007;102(8):1692-701. https://doi.org/10.1111/j.1572-0241.2007.01265.x

13. Wolters FL, Russel MG, Sijbrandij J, Ambergen T, Odes S, Riis L, Langholz E, Politi P, Qasim A, Koutroubakis I, Tsianos E, Vermeire S, Freitas J, van Zeijl G, Hoie O, Bernklev T, Beltrami M, Rodriguez D, Stockbrügger RW, Moum B. Phenotype at diagnosis predicts recurrence rates in Crohn's disease. Gut. 2006;55(8):1124-30. https://doi.org/10.1136/gut.2005.084061

14. Argüello M, Archila P, Sierra F, Otero W. Enfermedad inflamatoria intestinal. Rev Col Gastroentol. 1991;6(4):237-72.

15. Ka-Ho L, Hiu-Gong H, Chi-Ho N. Epidemiology and clinical characteristics of ulcerative colitis in Chinese population: Experience from a single center in Hong Kong. J Gastroenterol Hepatol. 2008;23(3):406-10. https://doi.org/10.1111/j.1440-1746.2007.05079.x

16. Langholz E, Munkholm P, Davidsen M, Binder V. Course of ulcerative colitis: analysis of changes in disease activity over year. Gastroenterology. 1994;107(1):3-11. https://doi.org/10.1016/0016-5085(94)90054-x

17. Larion S, Tavakoli H, Dececchis D, Arnold J, Vanslyke J. Psychiatric comorbidities in inflammatory bowel disease. Curr Psychiatry Rev. 2015;11(2):124-9. https://doi.org/10.2174/157340051102150502083333

18. Goodhand JR, Wahed M, Mawdsley JE, Farmer AD, Aziz Q, Rampton DS. Mood disorders in inflammatory bowel disease: Relation to diagnosis, disease activity, perceived stress, and other factors. Inflamm Bowel Dis. 2012;18(12):2301-9. https://doi.org/10.1002/ibd.22916

19. Trier Moller F, Andersen V, Andersson M, Jess T. Hospital admissions, biological therapy, and surgery in familial and 
sporadic cases of inflammatory bowel disease: A population-based cohort study 1977-2011. Inflamm Bowel Dis. 2015;21(12):2825-32. https://doi.org/10.1097/MIB.0000000000000545

20. Childers RE, Eluri S, Vazquez C, Weise RM, Bayless TM, Hutfless S. Family history of inflammatory bowel disease among patients with ulcerative colitis: A systematic review and meta-analysis. J Crohn's Colitis. 2014;8(11):1480-97. https://doi.org/10.1016/j.crohns.2014.05.008

21. Ng S, Tang W, Ching J. Incidence and phenotype of inflammatory bowel disease based on results from the Asia-Pacific Crohn's and colitis epidemiology study. Gastroenterology. 2013;145(1):158-65. https://doi.org/10.1053/j.gastro.2013.04.007

22. Lakatos L, Kiss LS, David G, Pandur T, Erdelyi Z, Mester G, Balogh M, Szipocs I, Molnar C, Komaromi E, Lakatos PL. Incidence, disease phenotype at diagnosis, and early disease course in inflammatory bowel diseases in Western Hungary, 2002-2006. Inflamm Bowel Dis. 2011;17(12):2558-65. https://doi.org/10.1002/ibd.21607

23. Nuij V, Zelinkova Z, Rijk M. Phenotype of inflammatory Netherlands: Bowel disease at diagnosis in the Delta, a population-based inception cohort study (the Delta Cohort). Inflamm Bowel Dis. 2013;19(10):2215-22. https://doi.org/10.1097/MIB.0b013e3182961626

24. Cantoro L, Di Sabatino A, Papi C, Margagnoni G, Ardizzone S, Giuffrida P, Giannarelli D, Massari A, Monterubbianesi R, Lenti MV, Corazza GR, Kohn A. The time course of diagnostic delay in inflammatory bowel disease over the last sixty years: An Italian multicentre study. J Crohns Colitis. 2017;11(8):975-80. https://doi.org/10.1093/ecco-jcc/jjx041

25. Lee DW, Koo JS, Choe JW, Suh SJ, Kim SY, Hyun JJ, Jung SW, Jung YK, Yim HJ, Lee SW. Diagnostic delay in inflammatory bowel disease increases the risk of intestinal surgery. World J Gastroenterol. 2017;23(35):6474-81. https://doi.org/10.3748/wjg.v23.i35.6474

26. Mitsuyama K, Niwa M, Takedatsu H, Yamasaki H, Kuwaki K, Yoshioka S, Yamauchi R, Fukunaga S, Torimura T. Antibody markers in the diagnosis of inflammatory bowel disease. World J Gastroenterol. 2016;22(3):1304-10. https://doi.org/10.3748/wjg.v22.i3.1304

27. Svoboda Baldin RK, Telles JEQ, Bonardi RA, Amarante $\mathrm{HMB}$ dos $\mathrm{S}$, Júnior AB. Do standardization and quantification of histopathological criteria improve the diagnosis of inflammatory bowel disease? J Bras Patol e Med Lab. 2014;50(3):221-8. https://doi.org/10.5935/1676-2444.20140019

28. Shi HY. Market share and costs of biologic therapies for inflammatory bowel disease in the USA. Aliment Pharmacol Ther. 2018;47(3):364-70. https://doi.org/10.1111/apt.14430

29. Romberg-Camps MJ, Dagnelie PC, Kester AD, Hesselinkvan de Kruijs MA, Cilissen M, Engels LG, Van Deursen C, Hameeteman WH, Wolters FL, Russel MG, Stockbrügger RW. Influence of phenotype at diagnosis and of other potential prognostic factors on the course of inflammatory bowel disease. Am J Gastroenterol. 2009; 104(2):371-83. https://doi.org/10.1038/ajg.2008.38

30. Solberg IC, Lygren I, Jahnsen J, Aadland E, Høie O, Cvancarova M, Bernklev T, Henriksen M, Sauar J, Vatn $\mathrm{MH}$, Moum B; IBSEN Study Group. Clinical course during the first 10 years of ulcerative colitis: results from a population-based inception cohort (IBSEN Study). Scand J Gastroenterol. 2009;44(4):431-40. https://doi.org/10.1080/00365520802600961

31. Bailey EH, Glasgow SC. Challenges in the medical and surgical management of chronic inflammatory bowel disease. Surg Clin North Am. 2015;95(6):1233-44. https://doi.org/10.1016/j.suc.2015.08.003 